City University of New York (CUNY) CUNY Academic Works

1990

\title{
A Kähler-Chern-Simons Theory and Quantization of Instanton Moduli Spaces
}

V. P. Nair

CUNY City College

Jeremy Schiff

Columbia University

\section{How does access to this work benefit you? Let us know!}

More information about this work at: https://academicworks.cuny.edu/cc_pubs/372

Discover additional works at: https://academicworks.cuny.edu

This work is made publicly available by the City University of New York (CUNY).

Contact: AcademicWorks@cuny.edu 
受入

$90-4-265$

勇工知闵書至
CU-TP-456

March 1990

\title{
A Kähler-Chern-Simons Theory and Quantization of Instanton Moduli Spaces
}

\author{
V.P.Nair and Jeremy Schiff \\ Physics Department, Columbia University \\ New York, NY 10027
}

\begin{abstract}
A five dimensional field theory is introduced which is an analogue of three dimensional Chern-Simons theory. The reduced phase space in the theory is a moduli space of instantons in four dimensional Euclidean gauge theory, with a symplectic structure induced by the Donaldson $\mu$-map. Issues related to quantization are discussed.
\end{abstract}

This research was supported in part by the U.S. Department of Energy. 
In this letter we discuss a five-dimensional field theory which generalizes certain features of the three-dimensional Chern-Simons theory (3d CST) which has recently attracted considerable interest [1,2]. Although the essential motivation in [1] was to provide an intrinsically 3-dimensional, quantum field theoretic interpretation of the Jones polynomials of links, which appear in 3d CST as correlation functions of Wilson loop operators, it also turned out that $3 \mathrm{~d}$ CST had a strong relationship with $2 \mathrm{~d}$ conformal field theory (2d CFT). Witten argued that $3 \mathrm{~d}$ CST on $\Sigma \times \mathbf{R}$, where $\Sigma$ is a Riemann surface, is an exactly solvable theory with a finite dimensional Hilbert space, which can be identified with the space of conformal blocks of an appropriate chiral algebra on $\Sigma$. These results have since been made explicit by a number of authors [3]. The constraint of gauge invariance on wave functionals is precisely the Ward identity for the currents of the Wess-Zumino-Witten (WZW) model on $\Sigma$; the wave functionals are thus the generating functionals for current correlators of the WZW model. The construction of link invariants emphasizes the topological nature of the theory (viz. the metric independence of the action). The existence of a chiral current algebra is however related to the fact that the reduced phase space is the space of flat gauge potentials on $\Sigma$ modulo gauge transformations; for gauge group $S U(N)$ this space can also be identified with the moduli space of stable, rank $N$ holomorphic vector bundles of Chern class zero over $\Sigma$, and holomorphic gauge transformations are naturally defined in this case. We wish to generalize this latter aspect.

There are a number of four-dimensional field theories for which properties analogous to those of $2 \mathrm{~d}$ CFT may be relevant. There are many supersymmetric gauge theories which are known to possess scale invariance, as exhibited by the vanishing of the $\beta$-function, the most famous example being the $N=4$ theory [4]. There are also indications that electrodynamics may have a phase transition at a critical coupling [5]; the theory at this point may have properties analogous to CFT's. A priori, looking for a five-dimensional analogue to $3 \mathrm{~d}$ CST may seem like a circuitous route to generalize the structure of $2 \mathrm{~d}$ CFTs to four dimensions. One could more directly attempt to generalize the infinite dimensional symmetry, the Virasoro algebra. Since this is related to invariance under complex analytic transformations, a natural generalization would be to use quaternionic analyticity (which is related to twistor holomorphicity via the Penrose transform). For $\mathbf{R}^{4}$ or $S^{4}$ the symmetry 
algebra becomes the direct sum of two Virasoro algebras of zero central charge [6], but the only field theories one can construct with this symmetry seem to be noninteracting [7]. Generalizing $3 \mathrm{~d}$ CST with its chiral algebra structure seems a more promising starting point.

The obvious guess for an appropriate five-dimensional theory would be one with an action based on the five-dimensional Chern-Simons term

$$
\begin{gathered}
S=\int_{M^{3}} \omega_{5}(A) \\
\omega_{5}=\frac{1}{24 \pi^{2}} \operatorname{Tr}\left(A(d A)^{2}+\frac{3}{2} A^{3} d A+\frac{3}{5} A^{5}\right)
\end{gathered}
$$

(wedge products have been suppressed here, and $A$ is a gauge potential on a five-manifold $M^{5}$ ). The abelian case of this has been analyzed by Floreanini et al [8], who have shown how to quantize the theory on $M^{4} \times \mathbf{R}$ and how to obtain from it a four-dimensional theory with a $U(1)$ current algebra. In the non-Abelian case the action exists only for gauge groups $S U(N), N \geq 3$ and $S O(6)$. There is no simple chiral algebra structure and the reduced phase space is not of immediate interest, so even though it seems likely that from a quantum field theory based on this action it might be possible to derive some interesting results, including possibly an understanding of higher dimensional knot invariants, we do not study it here.

Instead we shall consider the following theory: we take spacetime to be a Kähler manifold $M^{4}$ and denote the Kähler form on $M^{4}$ by $\omega$. (Analogy with Riemann surfaces in two dimensions would suggest the use of compact Kähler manifolds. Our calculations show no pathologies for $\mathbf{R}^{4}$ and there seems to be no reason to exclude this simple case.) The action is taken to be

$$
S=-\frac{k}{4 \pi} \int_{M^{4} \times \mathbf{R}} \operatorname{Tr}\left(\left(A \wedge d A+\frac{2}{3} A \wedge A \wedge A\right) \wedge \omega+\phi \wedge F+\phi^{*} \wedge F\right)
$$

Here $A$ is the locally defined Lie algebra valued 1-form on $M^{4} \times \mathbf{R}$ corresponding to a gauge potential or a connection on a principal $G$-bundle over $M^{4} \times \mathbf{R}$. ( $G$ is a semisimple, compact Lie group; for all calculations we will take $G=S U(2))$. $F$ is the associated field strength, $F=d A+A \wedge A . \phi, \phi^{*}$ are, respectively, locally defined Lie algebra valued $(2,0)$ 
and $(0,2)$ forms on $M^{4}$, and also 1 -forms on $\mathbf{R}$, i.e. if $z^{i}, i=1,2$ are complex coordinates on $M^{4}$ and $t$ is the coordinate on $\mathbf{R}$, then in local coordinates

$$
\begin{gathered}
\phi=\phi\left(z^{i}, \bar{z}^{i}, t\right) d z^{1} \wedge d z^{2} \wedge d t \\
\phi^{*}=\phi^{*}\left(z^{i}, \bar{z}^{i}, t\right) d \bar{z}^{1} \wedge d \bar{z}^{2} \wedge d t
\end{gathered}
$$

Gauge transformations act upon the fields as follows:

$$
\begin{gathered}
A \rightarrow A^{g}=g A g^{-1}+g d g^{-1} \\
\phi \rightarrow g \phi g^{-1} \quad, \quad \phi^{*} \rightarrow g \phi^{*} g^{-1}
\end{gathered}
$$

where $g$ is a Lie group valued function on $M^{4} \times \mathbf{R}$. Invariance of the action under homotopically trivial gauge transformations follows from the fact that $\omega$, being a Kähler form on $M^{4}$, and having no $t$ dependence, is closed on $M^{4} \times \mathbf{R}$. In general, for compact $M^{4}$, there will exist homotopically nontrivial gauge transformations and invariance of $e^{i S}$ under these will require $k$ to be an integer. In local coordinates we can write $\omega=\frac{1}{2} \omega_{\mu \nu} d x^{\mu} \wedge d x^{\nu}$, and we take $\omega$ throughout to be self-dual (with the understanding that similar results exist if we take $\omega$ to be anti-self-dual). The theory we have described is not topological, because of our use of the Kähler strcture on $M^{4}$. In fact our assumption that $M^{4}$ is Kähler is a little stronger than is necessary, and many of our results go through when $M^{4}$ is any complex manifold and $\omega$ is an arbitrary symplectic form on $M^{4}$. We shall keep the assumption that $M^{4}$ is Kähler, though, and we refer to this theory as a Kähler-Chern-Simons theory (KCST); we shall see that despite the lack of topological invariance, the theory has many features common with $3 \mathrm{~d}$ CST.

Consider now the canonical quantization of (2). It is convenient to treat the "time" component $A_{t}$ separately from the space components $A_{\mu}, \mu=1,2,3,4$. We need to impose the constraints $\Pi_{A_{t}}=0, \Pi_{\phi}=0, \Pi_{\phi^{*}}=0$. These are all first class; we can eliminate them by setting $A_{t}=0, \phi=0, \phi^{*}=0$. We must further impose the corresponding equations of motion

$$
\begin{gathered}
F \wedge \omega=0 \\
F^{(2,0)}=F^{(0,2)}=0 .
\end{gathered}
$$


$(5 a)$ is the Gauss law of the theory. The remaining equations (for $A_{\mu}$ ) in (2) imply that the time-derivative of $A_{\mu}$ is zero; there is no real dynamics, only the structure of the Hilbert space is relevant. The symplectic 2 -form following from (2) is

$$
\Omega=\frac{k}{4 \pi} \int_{M^{4}} \operatorname{Tr}(\delta A \wedge \delta A) \wedge \omega
$$

where $\delta$ denotes the exterior derivative on $\mathcal{A}$, the space of gauge potentials on $M^{4}$. The corresponding Poisson bracket for $A_{\mu}(x)$ is

$$
\left[A_{\mu}^{a}(x), A_{\nu}^{b}(y)\right]=\frac{4 \pi}{k} \frac{\omega_{\mu \nu}}{\sqrt{g}} \delta^{(4)}(x-y) \delta^{a b}
$$

(here we take $\operatorname{Tr}\left(T^{a} T^{b}\right)=-\frac{1}{2} \delta_{a b}$, where $T^{a}$ are the generatös we use for the Lie algebra).

As in $3 \mathrm{~d}$ CST there are two ways to proceed: one can quantize, using (7), and then impose the constraints (5) on the states; alternatively one can solve the constraints and then quantize on the reduced phase space (the space of solutions of the constraints, modulo the remaining gauge invariance) [9]. For the most part we shall follow the latter approach. It is well known that the equations (5) express the vanishing of the self-dual part of $F$ on a complex manifold [10]. The solutions are thus anti-self-dual instantons, and the reduced phase space is the moduli space of instantons. (If we take $\omega$ to be anti-self-dual, we would obtain the space of self-dual instantons. Note that unlike in 3d CST, where the reduced phase space consists of flat connections on a Riemann surface and hence zero topological charge, in this theory there is no constraint on the magnitude of the appropriate topological charge, the second Chern number.) The quantization of our theory reduces to quantization of moduli spaces of instantons with the symplectic structure (6) (the gauge invariance of $\Omega$ will be examined shortly).

Symmetry structure of $3 d$ CST and 5d KCST. As mentioned above, in 3d CST the reduced phase space is the space of flat gauge potentials on a given Riemann surface $\Sigma$, modulo gauge transformations. This space can be identified in two ways. We can solve the constraint $F=0$ to write, locally, $A=u d u^{-1}$. Then on the set of $u$ 's so defined we further have the action of gauge transformations. The reduced phase space is thus the set of $u$ 's 
modulo $\mathcal{G}_{\Sigma}$, the group of gauge transformations on $\Sigma$. Alternatively, one can parametrise $A_{\bar{z}}$ locally as $U \partial_{\bar{z}} U^{-1}$ where $U$ is $G^{\mathbf{C}}$ valued. $F=0$ then constrains the hermitian part of $U$ and the action of dividing out by $\mathcal{G}$ removes the unitary part. In fact this two step reduction can be achieved by dividing out by $\mathcal{G}^{\mathbf{C}}$, the group of complexified gauge transformations. Thus one can identify the reduced phase space as $\mathcal{A}_{\Sigma} / \mathcal{G}_{\Sigma}^{\mathrm{C}}$, where $\mathcal{A}_{\Sigma}$ denotes the space of gauge potentials on $\Sigma$. In other words Hamiltonian or symplectic reduction by $F=0$ is equivalent to dividing out by $\mathcal{G}_{\Sigma}^{\mathrm{C}}[1,11]$. The significance of this is that if we quantize 3d CST before imposing the Gauss law of the theory, we get a set of states which have a natural $\mathcal{G}_{\Sigma}^{\mathrm{C}}$ action on them; this is the chiral Kac-Moody symmetry. The description in terms of the $U$ 's shows further that the phase space is also the moduli space of stable, rank $N$ (for gauge group $S U(N)$ ) holomorphic vector bundles of Chern class zero over $\Sigma$, a natural focus of interest in topology [1,11]. (In the above we assume that before dividing out the space of gauge potentials by the group of gauge transformations, we remove certain types of potentials that do not give rise to stable bundles. For details see [11]. Similar provisos apply in the next paragraph.)

The phase space of KCST, the moduli space of instantons of given 2nd Chern number on $M^{4}$, has analogous interpretations. First, if we denote by $\mathcal{A}^{(1,1)}$ the set of gauge potentials on $M^{4}$ such that $F^{(2,0)}=F^{(0,2)}=0$, and by $\mathcal{G}^{\mathrm{C}}$ the group of complexified gauge transformations on $M^{4}$, then the phase space is obtained by symplectic reduction of $\mathcal{A}^{(1,1)}$ by the Gauss law (5a). This is also the space $\mathcal{A}^{(1,1)} / \mathcal{G}^{\mathrm{C}}$. Thus if we quantize the theory before imposing the Gauss law $(5 a)$ we would get a set of states with a natural $\mathcal{G}^{\mathrm{C}}$ action on them, a four-dimensional analogue of Kac-Moody symmetry or more precisely a chiral symmetry algebra. (In the case of instantons on $\mathbf{R}^{4}$ this symmetry has been known for some time [12].) The conditions (5b) also show that we can write locally $A_{\bar{z}_{i}}=U \partial_{\bar{z}_{i}} U^{-1}, \quad i=1,2$. Donaldson's theorem [13] tells us that there is a unique solution to $(5 a)$ for each solution to $(5 b)$ and hence the phase space can also be identified as the moduli space of stable, rank $N$ (for gauge group $S U(N)$ ), holomorphic vector bundles over $M^{4}$. Thus we have a close analogue to $3 \mathrm{~d}$ CST. The observables in KCST correspond to (functions of) the instanton moduli; these are the analogues of the holonomies in $3 \mathrm{~d}$ CST. 
Some of the current algebraic aspects of the theory are revealed by considering quantization before reduction for the case of zero instanton number. For compact $M^{4}$, using the parametrization $A_{\bar{z}_{i}}=U \partial_{\bar{z}_{i}} U^{-1}$, one can see that the wavefunction satisfying (5a) is $\psi=\exp (k \mathcal{S})$ where

$$
\mathcal{S}=\frac{1}{8 \pi} \int_{M^{4}} g^{i j} \operatorname{Tr}\left(\partial_{i} U \partial_{j} U^{-1}\right)+\frac{i}{6 \pi} \int_{\tilde{M}} \operatorname{Tr}\left(U^{-1} d U\right)^{3} \wedge \omega
$$

Here $\tilde{M}$ is taken to be $M^{4} \times[0,1]$; we identify one boundary component of this with our original $M^{4}$, and extend $U$ into $\tilde{M}$ in such a way that it tends to some fixed function on the other component of the boundary (determined by the homotopy class of $U$ on the original $M^{4}$ ). This expression $\mathcal{S}$ is the analogue of the action for the WZW model (with complex $U$ ) which determines the wavefunction for $3 \mathrm{~d}$ CST. There is a Polyakov-Wiegmann type formula

$$
\begin{gathered}
\mathcal{S}\left(U_{1} U_{2}\right)=\mathcal{S}\left(U_{1}\right)+\mathcal{S}\left(U_{2}\right)+\Gamma\left(U_{1}, U_{2}\right) \\
\Gamma\left(U_{1}, U_{2}\right)=\frac{1}{2 \pi} \int_{M^{4}} g^{i j} \operatorname{Tr}\left(U_{1}^{-1} \partial_{i} U_{1} U_{2} \partial_{j} U_{2}^{-1}\right)
\end{gathered}
$$

and there are conserved chiral currents. Many aspects of the two-dimensional WZW model will generalize and we will have a chiral algebra structure [12]. The normalization integral for $\psi$ will involve $\exp (k S(H))$, where $H$ is the hermitian matrix $U^{\dagger} U$; the variational equation for $\mathcal{S}(H)$ is exactly the equation $F \wedge \omega=0$. Thus $\mathcal{S}$ is an action for the self-dual gauge theory. Various authors [14] have written alternate actions for the $S U(2)$ self-dual gauge theory in terms of a parametrization of the matrix $U$.

Gauge invariance of the symplectic form. The symplectic form (6) is invariant, as a 2form, on the space of potentials satisfying $F \wedge \omega=0$, under the gauge transformation given locally by (4). It is straightforward to check that

$$
\begin{gathered}
\Omega\left(A^{g}\right)-\Omega(A)=\delta\left(k \Omega^{1}(A, g)\right) \\
\Omega^{1}(A, g)=\frac{1}{2 \pi} \int_{M^{4}} \operatorname{Tr}\left(g^{-1} \delta g F\right) \wedge \omega
\end{gathered}
$$

Thus with the condition (5a), e.g. on the space of instanton solutions, $\Omega$ is gauge invariant. We can in fact say one thing more. Although (6) is not invariant under the transformation 
(4) on the whole space $\mathcal{A}$, it defines a (non-trivial) element of the second cohomology $H^{2}(\mathcal{A} / \mathcal{G})$, since the $\Omega^{1}(A, g)$ obeys a cocycle condition $\Omega^{1}(A, h g)=\Omega^{1}(A, g)+\Omega^{1}\left(A^{g}, h\right)$. One can further check that the cohomology class of $\Omega$ in $\mathcal{A} / \mathcal{G}$ depends only on the class of $\omega$ in $H^{2}\left(M^{4}\right)$. To do this, note we can write (6) in the form

$$
\Omega=\frac{k}{4 \pi} \int_{M^{4}} \operatorname{Tr}(\mathcal{F} \wedge \mathcal{F}) \wedge \omega
$$

where

$$
\mathcal{F}=(d+\delta) A+A \wedge A
$$

(the extra terms do not contribute to the integral). If we change $\omega$ by $d \alpha$, where $\alpha$ is a 1 -form on $M^{4}$, then since $\delta \alpha=0$ and $(d+\delta) \operatorname{Tr}(\mathcal{F} \wedge \mathcal{F})=0$ we see that $\Omega$ changes by a term proportional to

$$
\delta\left(\int_{M^{4}} \operatorname{Tr}(\mathcal{F} \wedge \mathcal{F}) \wedge \alpha\right)
$$

the term in parentheses defines a 1 -form on $\mathcal{A} / \mathcal{G}$. Thus we have a map from $H^{2}\left(M^{4}\right)$ to $H^{2}(\mathcal{A} / \mathcal{G})$. This is exactly the Donaldson $\mu$-map [15], in the cohomology version discussed by Braam and multiplied by a factor of $2 \pi k$ since (6) was derived from an action, which is measured naturally in units of $2 \pi$. If we consider the 2 -form derived from $T$, where $S=2 \pi T$, we would obtain the Donaldson map times $k$; the requirement that $k$ is an integer is equivalent to the requirement that we obtain a map from $H^{2}\left(M^{4}, \mathbf{Z}\right)$ to $H^{2}(\mathcal{A} / \mathcal{G}, \mathbf{Z})$.

The Donaldson $\mu$-map is expected to be of interest in Euclidean gauge theories. The configuration space of gauge theories is $\mathcal{A} / \mathcal{G}$; the $\mu$-map is very useful in understanding the topology of this space. The natural appearance of the $\mu$-map in our theory suggests that this theory may also provide a probe of some topological features of Euclidean gauge theory.

We shall now consider the quantization of the reduced phase space for some special cases. In general, the solutions to the equations (5) will include instantons of any topological charge, i.e. the magnitude of the Chern number is arbitrary. The Hilbert space is thus characterized by $k$ and the instanton number $q$. Eventually if one is to interpret the results in a way similar to the WZW model in two dimensions, we need a rule for comparing different values of $q$. It is not clear at present how one might do this, but the 
existence of Bäcklund transformations that change instanton numbers [16] suggests that this problem is solvable; i.e. one may eventually be able to construct a 'universal moduli space' which includes all values of $q$. For our calculations we shall consider moduli spaces for fixed $q$.

Calculations on $\mathbf{R}^{4}$. By instanton moduli spaces for $\mathbf{R}^{4}$ we really mean instanton moduli spaces for $S^{4}$; because of the conformal invariance of the self-duality equations in four dimensions, every instanton on $S^{4}$ is an instanton on $\mathbf{R}^{4}$ (we assume the standàrd Euclidean metrics here). We choose to work on $\mathrm{R}^{4}$ rather than $S^{4}$ since otherwise we lose the Kähler structure.

The general self-dual 2 -form on $\mathbf{R}^{4}$ is given by $\dot{\omega}=\omega^{i} \eta_{\mu \nu}^{i} d x^{\mu} \wedge d x^{\nu}$, where $\eta_{\mu \nu}^{i}$ are the 't Hooft $\eta$-tensors (see [17] for properties). The general 1-instanton solution has five parameters, a vector $a^{\mu}$ and a positive scalar $\lambda$, corresponding to the position and size, and is given by [18]

$$
A_{\mu}^{i}=\frac{2 \bar{\eta}_{\mu \nu}^{i}(x-a)^{\nu}}{(x-a)^{2}+\lambda^{2}}
$$

(in this section we use the basis $\sigma^{i} / 2 i$ for Lie algebra of $S U(2)$, where $\sigma^{i}$ are the Pauli sigma matrices) The $q$-instanton solution has $8 q-3$ parameters; the solutions can be constructed explicitly [19] but are very complicated. The general 2 -instanton solution has a simple form [20]

$$
\begin{gathered}
A_{\mu}^{i}=-\eta_{\mu \nu}^{i} \partial_{\nu} \ln \rho \\
\rho=\frac{\lambda_{1}^{2}}{\left(x-a_{1}\right)^{2}}+\frac{\lambda_{2}^{2}}{\left(x-a_{2}\right)^{2}}+\frac{\lambda_{3}^{2}}{\left(x-a_{3}\right)^{2}}
\end{gathered}
$$

Here $\lambda_{1}, \lambda_{2}, \lambda_{3}$ are positive scalars, but only two of them are physical (dividing $\rho$ by an overall constant does not affect $A$ ); $a_{1}, a_{2}, a_{3}$ are vectors, but there is one superfluous parameter (see [20]). In fact, for $q \geq 3$, a $5 q+4$ parameter subfamily of the $8 q-3$ parameter family of instantons has form (13a), with

$$
\rho=\sum_{r=1}^{q+1} \frac{\lambda_{r}^{2}}{\left(x-a_{r}\right)^{2}}
$$


in the obvious notation (again only $q$ of the $q+1$ parameters $\lambda_{1}, \lambda_{2}, \ldots, \lambda_{q+1}$ are physical). Note that the $q \geq 2$ solutions we have given above all have apparent singularities, but in fact these are gauge artefacts.

For any $q$ we wish to compute the symplectic form (6) on the instanton moduli space. The first thing we notice is that for the solutions we have given above $A$ appears to go like $1 /|x|$ for large $|x|$; again this is a gauge artefact, and in an appropriate gauge $A$ goes like $1 /|x|^{2}$. Thus it would seem (6) will have logarithmic divergences. In fact explicit calculations show that this is not the case (at least for the $q=1$ and $q=2$ spaces, and the $5 q+4$ dimensional subspace described above of the general $q \geq 3$ space). This remarkable fact emerges in calculations from some seemingly coincidental cancellations of terms; we currently have no insight into this result.

For $q=1$ (where topologically the moduli space is $\mathbf{R}^{4} \times(0, \infty)$ ), it is a straightforward matter to calculate $\Omega$ (using $\eta$-tensor identities from [17]) to find that

$$
\Omega=\frac{3 k \pi}{4} \omega_{\mu \nu} \delta a^{\mu} \wedge \delta a^{\nu}
$$

It is straightforward to apply the techniques of geometric quantization to this. $\lambda$ plays no role, i.e. quantization can be done for each value of $\lambda$. Choosing a polarization amounts to choosing two appropriate linear combinations of the $a^{\mu}$. We can write

$$
\omega_{\alpha \dot{\alpha} \beta \dot{\beta}}=\omega_{\mu \nu} \sigma_{\alpha \dot{\alpha}}^{\mu} \sigma_{\beta \dot{\beta}}^{\nu}=\left(\pi_{\dot{\alpha}} \bar{\pi}_{\dot{\beta}}+\pi_{\dot{\beta}} \bar{\pi}_{\dot{\alpha}}\right) \epsilon_{\alpha \beta}
$$

where we use the usual spinor notation, i.e. $\sigma_{\alpha \dot{\alpha}}^{\mu}=\left(1, i \sigma^{i}\right)$ and $\bar{\pi}_{\dot{\alpha}}=\epsilon_{\dot{\alpha} \dot{\beta}}\left(\pi_{\dot{\beta}}\right)^{*}$, the star denoting complex conjugation. The two component spinor $\pi_{\dot{\alpha}}, \dot{\alpha}=1,2$, characterizes the choice of complex structure on $\mathbf{R}^{4}$. Introducing the spinor version of $a^{\mu}$ by $a^{\mu}=\sigma_{\alpha \dot{\alpha}}^{\mu} V^{\alpha \dot{\alpha}}$ and $z^{\alpha}=V^{\alpha \dot{\alpha}} \pi_{\dot{\alpha}}$ we get

$$
\Omega=\frac{3 k \pi}{2} \epsilon_{\alpha \beta} d \bar{z}^{\alpha} \wedge d z^{\beta}
$$

The wave functions are thus given, up to normalization, in the obvious holomorphic polarization by

$$
\psi_{n m}=z_{1}^{n} z_{2}^{m}
$$

with the standard Bargmann inner product induced by the Kähler potential $K=\frac{3 k \pi}{2} \epsilon_{\alpha \beta} \bar{z}^{\alpha} z^{\beta}$. Note that the states $\psi_{n m}$ for $n+m=2 j$ provide a spin $j$ representation of $S U(2)$; by our 
choice of $\omega$ we have broken Lorentz invariance to $S U(2)$, so we expect our states to give representations of $S U(2)$, and we see that each representation appears precisely once.

The logical next step would be to understand whether the 2- and multi-instanton Hilbert spaces differ significantly from the tensor products of 1-instanton Hilbert spaces. The calculation of $\Omega$ for the 2-instanton solution is very involved and we have to consider some sort of approximation. The 13 parameters in the solution (13) should correspond to the two positions (eight parameters), two size parameters and three parameters of relative group orientation for the two instantons. In the limit of $\lambda_{2}^{2} / \lambda_{1}^{2}, \lambda_{3}^{2} / \lambda_{1}^{2} \ll 1$ and large $\left|a_{2}-a_{3}\right|$ one can see that the 2 -instanton solution behaves approximately as two 1 -instantons with centers at $a_{2}$ and $a_{3}$. We shall do the calculation in this 'dilute gas' limit. In terms of the function $\rho$ of equations (13), $\Omega$ can be written as

$$
\Omega=\frac{k}{8 \pi} \int_{M^{4}} \omega_{\mu \nu} \frac{\rho \delta \rho_{\mu}-2 \rho_{\mu} \delta \rho}{\rho^{3}} \wedge \delta \rho_{\nu}
$$

The probelmatic factor in performing the integration exactly is the $\rho^{3}$ factor in the denominator. The integral is manageable if we expand the $\rho^{-3}$ factor in powers of $\lambda_{2}^{2} / \lambda_{1}^{2}$ and $\lambda_{3}^{2} / \lambda_{1}^{2}$. Thus in a 'dilute gas' limit we expect to get

$$
\Omega=\omega_{\mu \nu}\left(\delta a_{2}^{\mu} \wedge \delta a_{2}^{\nu}+\delta a_{3}^{\mu} \wedge \delta a_{3}^{\nu}\right)+\text { corrections }
$$

Furthermore, in this limit we can identify the angular components of the transiationinvariant combination $a_{1}-\frac{1}{2}\left(a_{2}+a_{3}\right)$ as corresponding to relative group orientation between two distant instantons; it is therefore interesting to calculate the coefficient of $\delta a_{1}^{\mu} \wedge a_{1}^{\nu}$ occuring in the 'correction' terms of (20). So far we have calculated these terms (using the expansion procedure explained above) to lowest order in $\lambda_{2}^{2} / \lambda_{1}^{2}$ and $\lambda_{3}^{2} / \lambda_{1}^{2}$, obtaining the result zero. The Hilbert space for the 2 -instanton case is thus a tensor product of two 1-instanton Hilbert spaces even up to first order corrections in $\lambda_{2}^{2} / \lambda_{1}^{2}$ and $\lambda_{3}^{2} / \lambda_{1}^{2}$.

Remarks on $\mathbf{C} P^{2}$. We shall now briefly consider aspects of the relevant moduli spaces for the case of $C P^{2}$. The construction of holomorphic rank 2 vector bundles on $\mathrm{C} P^{2}$ is known [21], and is analogous to the construction of vector bundles on $\mathbf{C} P^{3}$ used to obtain instantons on $S^{4}[19]$. Bundles are classified by the frst two Chern numbers $c_{1}, c_{2}$. 
For example, for $\left(c_{1}, c_{2}\right)=(0, q)$, the complex dimension of the moduli space of vector bundles is $4 q-3$. While Donaldson's theorem [13] guarantees the existence of anti-selfdual instantons for stable vector bundles, there is no general procedure for constructing the potentials given the vector bundle. For $q=1$ there are no stable vector bundles and hence there is no 1 -instanton solution [22]. (There is a 5-parameter self-dual 1-instanton solution on $\mathrm{C} P^{2}$ [23], which is, however, not of relevance to us.) For $q \geq 2$, stable vector bundles, and hence $q$-instantons, are known to exist [22]. $\Omega$ will give us a Kähler structure on the moduli spaces; the Kähler metric induced on the moduli space is expected to be the natural Riemannian metric on $\mathcal{A} / \mathcal{G}$ restricted to the moduli space [24]. We hope to pursue these matters further elsewhere.

Discussion. An interesting feature of the theory is the emergence of the $\mu$-map. This may play an important role in understanding Euclidean gauge theories, as it provides a symplectic structure on the set of solutions to a Euclidean gauge theory. For field theories on $\mathbf{R}^{4}$, for example, there is the possibility of performing radial quantization. The symplectic form, for standard Yang-Mills action, is given by

$$
\delta\left(\int d \Sigma^{\mu} F_{\mu \nu}^{i} \delta A_{\nu}^{i}\right)
$$

where the integral is over a 3-sphere centered at the origin. A straightforward calculation shows that this vanishes for the 1-instanton moduli space. Quantization with $\Omega$, however, can still be carried out. In this context, it is important to identify the complete set of finite action solutions of the Yang-Mills equations. Equations (5) are appropriate if all the solutions are instantons. This is not the case for $S^{4}$ [25]. It would be interesting if other solutions could be associated with relaxing the conditions $F^{(0,2)}=F^{(2,0)}=0$, which have been imposed in a somewhat ad hoc fashion in our theory. (Notice also that for $\mathbf{R}^{4}$, equations (5) can be written as $F \wedge \omega^{i}=0$, where $\omega^{i}=\eta_{\mu \nu}^{i} d x^{\mu} \wedge d x^{\nu}$. Ideally we would like some way to avoid choosing a distinguished complex structure.)

In addition to the connection of KCST to gauge theories, the four-dimensional currentalgebraic aspects of KCST look very promising. We hope to be able to make explicit the interesting properties of the action defined by (8); specifically we note that since it is in 
some sense integrable, we might expect the quantum theory to be renormalizable and even possibly finite.

Acknowledgements. We thank Michiel Bos and Peter Woit for discussions and a critical reading of the manuscript. We also thank P. Feehan, R. Friedman and J. Morgan for discussions.

\section{References}

[1] E.Witten, Comm.Math.Phys. 121 (1989) 351.

[2] S.Deser, R.Jackiw and S.Templeton, Phys.Rev.Lett. 48 (1982) 975; Ann.Phys. 140 (1982) 372; J.Schonfeld, Nucl.Phys. B185 (1981) 157; for an overview of different uses of the Chern-Simons term see R.Jackiw, Topics in Planar Physics, MIT preprint CTP\# 1824 (Dec 1989).

[3] M.Bos and V.P.Nair, Phys.Lett. B223 (1989) 61; Int.J.Mod.Phys A5 (1990) 959; S.Elitzur, G.Moore, A.Schwimmer and N.Seiberg, Nucl.Phys. B326 (1989) 108; J.M.F.Labastida and A.V.Ramallo, Phys.Lett. B227 (1989) 92; H.Murayama, Tokyo preprint UT-542-TOKYO (1989); A.P.Polychronakos, University of Florida preprints UFIFT-HEP-89-7, UFIFT-HEP-89-9; T.R.Ramadas, I.M.Singer and J.Weitsman, MIT Math Dept. preprint.

(4) For the $N=4$ theory see L.Brink, O.Lindgren and B.Nilsson, Phys.Lett. B123 (1983) 323; P.Howe, K.Stelle and P.K.Townsend, Phys.Lett. B124 (1983) 55; S.Mandelstam, Nucl.Phys. B213 (1983) 149. For other finite supersymmetric gauge theories see P.S.Howe, K.S.Stelle and P.C.West, Phys. Lett. 124B (1983) 55; O.Piguet and K.Sibold, Phys.Lett. B177 (1986) 373; D.I.Kazakov, Phys.Lett. B179 (1986) 352; X.Jiang and X.Zhou, Phys.Lett. B197 (1987) 156.

[5] V.A.Miransky, Nuov.Cim 90A (1985) 149; P.I.Fomin, V.P.Gusynin, V.A.Miransky and Yu.A.Sitenko, Riv.Nuov.Cim. 6 (1983) No.5; D.K.Hong, S.G.Rajeev, Rochester University preprint UR-1135, ER-13065-597.

[6] F.Gürsey and H.C.Tze, Lett.Math.Phys. 8 (1984) 387; F.Gürsey and V.P.Nair, un- 
published.

[7] Y.Eisenberg, Phys.Lett. B233 (1989) 349.

[8] R.Floreanini and R.Percacci, Phys.Lett. B224 (1989) 291; R.Floreanini, R.Percacci and R.Rajaraman, Phys.Lett. B231 (1989) 119.

[9] These two procedures may not in general be equivalent. See G.V.Dunne, R.Jackiw, C.A.Trugenberger Ann.Phys. 194 (1989) 197; G.Zemba, Int.J.Mod.Phys. A5 (1990) 559.

[10] M.F.Atiyah, Geometry of Yang-Mills Fields (Scuola Normale Superiore, Pisa, 1979); C.N.Yang, Phys.Rev.Lett. 38 (1977) 1377.

[11] M.F.Atiyah and R.Bott, Phil.Trans.R.Soc.Lond A308 (1982) 523; N.J.Hitchin, A.Karlhede, U.Lindström and M.Rocek, Comm.Math.Phys. 108 (1987) 535.

[12] See for example L.Dolan, Phys.Rep. 109 (1983) 1; L.-L.Chau, UC Davis preprint, UCD-87-38.

[13] S.K.Donaldson, Proc.Lond.Math.Soc.(3) 50 (1985) 1.

[14] K.Pohlmeyer, Comm:Math.Phys. 72 (1980) 37; H.J.de Vega, Comm.Math.Phys. 116 (1988) 659; R.S.Ward, Nonlinearity 1 (1988) 671.

[15] S.K.Donaldson, J.Diff.Geom. 24 (1986) 275; P.Braam, lectures given at the Fifth Annual University of California Summer School on Nonlinear Science, Physics and Geometry (June 1989).

[16] E.F.Corrigan, D.B.Fairlie, P.Goddard and R.G.Yates, Comm.Math.Phys. 58 (1978) 223.

[17] G.'t Hooft, Phys.Rev. D14 (1976) 3432.

[18] A.A.Belavin, A.M.Polyakov, A.S.Schwartz and Yu.S.Tyupkin, Phys.Lett. 59B (1975) 85. For a general reference on instantons in physics see R.Rajaraman, Solitons and Instantons (North Holland, Amsterdam, 1982).

[19] M.F.A tiyah, V.G.Drinfeld, N.J.Hitchin and Yu.I.Manin, Phys.Lett. 65A (1978) 185; N.H.Christ, N.K.Stanton and E.J.Weinberg, Phys.Rev. D18 (1978) 2013.

[20] R.Jackiw, C.Nohl and C.Rebbi, Phys.Rev. D15 (1977) 1642.

[21] C.Okonek, M.Schneider and H.Spindler Vector Bundles on Complex Projective Spaces (Birkhäuser, Boston, 1980). 
[22] C.H.Taubes J.Diff. Geom 19 (1984) 517.

[23] S.K.Donaldson in Geometry Today, Progress in Math No. 60 (Birkhäuser, Boston, 1985); N.P.Buchdahl, J.Diff.Geom. 24 (1986) 19.

[24] H.Doi, Y.Matsumoto and T.Matumoto in A Fete of Topology (Academic Press, New York, 1988); D.Groisser and T.H.Parker, Comm.Math.Phys. 112 (1987) 663; J.Diff. Geom. 29 (1989) 499; K. Kobayashi, Hiroshima Math.J. 19 (1989) 243; D.Groisser, preprint, to appear in Invent.Math.; S.K.Donaldson in The Interface of Mathematics and Particle Physics (Oxford University Press, Oxford, 1990).

[25] G.Sibner, R.J.Sibner and K.Uhlenbeck, Proc.Natl.Acad.Sci.USA 86 (1989) 8610; G.Bor and R.Montgomery, "SO(3) Invariant Yang-Mills Fields which are not self-dual" preprint. 